\title{
Carotid Web: Appearance at MR Angiography
}

I $\mathrm{n}$ their article entitled "Carotid Webs and Recurrent Ischemic Strokes in the Era of CT Angiography," Choi et al ${ }^{1}$ described the prevalence, demographics, clinical presentation, imaging features, histopathology, and stroke risk associated with carotid webs. We read this thorough review with great interest, and it has already helped us in our clinical practice.

Recently, an otherwise healthy 49-year-old woman presented to our hospital with neck pain and left-sided numbness, prompting an imaging work-up, including CTA of the head and neck. On sagittal reformatted images (Fig $1 A$ ), we noticed a thin intraluminal filling defect along the posterior wall of the carotid bulb, which appeared as a septum on axial images (Fig 2A). When we used the definition set forth by Choi et al, ${ }^{1}$ our patient had findings consistent with a carotid web. No other abnormality was identified on her CTA. The carotid web was ipsilateral to the patient's clinically diagnosed TIA. Work by Choi et al and and Morgenlander and Goldstein $^{2}$ has demonstrated an association between TIA and ipsilateral carotid webs and has provided evidence to support the diagnosis in this patient.

Choi et $\mathrm{al}^{1}$ mentioned that the MR imaging appearance of carotid webs has not been reported in the literature. Our patient also underwent MRA of the neck during her hospitalization, which included 2D time-of-flight and a black-blood, axial T1weighted fat-suppressed sequence for the evaluation of vessel wall pathology. On the T1-weighted fat-saturated images, we observed a crescentic hyperintense signal (Fig $2 A$ ) consistent with hemorrhage. ${ }^{3,4}$ The finding of Choi et $\mathrm{al}^{1}$ of hemorrhage in 2 of the 4 patients for which carotid endarterectomy specimens were available is consistent with our findings on T1-weighted black-blood imaging in this patient. Axial source images from the 2D TOF image (Fig 2B) demonstrated abnormal flow-related enhancement in the area of the known web, possibly due to diminished or turbulent flow. The significance of abnormal flow in carotid webs in the pathogenesis of ipsilateral brain ischemia is uncertain but may be explained by the hypothesis of Choi et $\mathrm{al}^{1}$ that "the existence of turbulence and stasis in a cul-de-sac upstream to the web...could potentially create a thrombogenic milieu."

Our case also demonstrates that when evaluated without CTA,

http://dx.doi.org/10.3174/ajnr.A4598

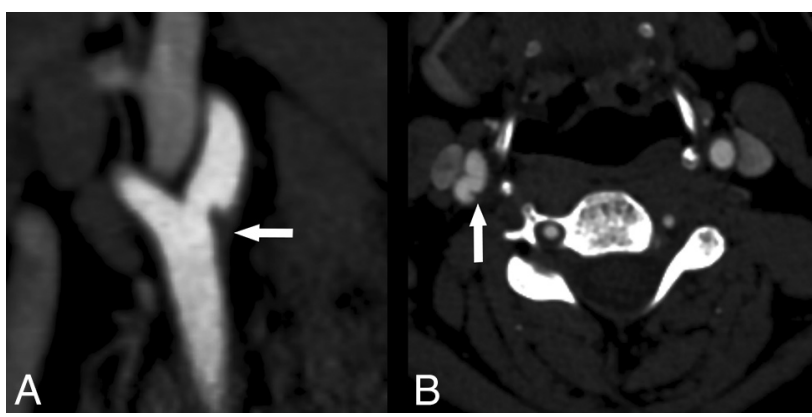

FIG 1. Sagittal reformat (A) from a CT angiogram demonstrates a thin intraluminal filling defect (arrow) along the posterior wall of the carotid bulb just beyond the carotid bifurcation. Axial source image $(B)$ demonstrates a thin posterior septum (arrow) projecting into the lumen of the proximal right internal carotid artery. This finding is consistent with a carotid web using the definition set forth by Choi et al.'

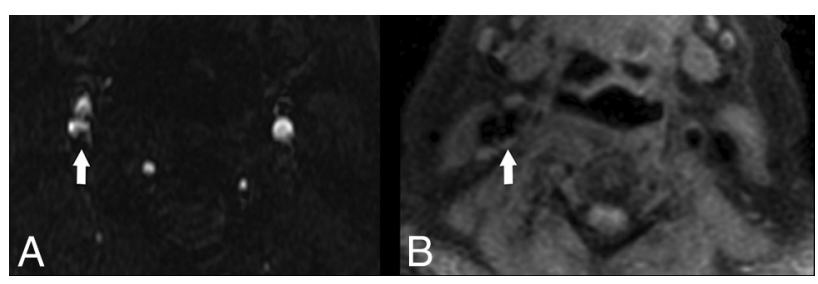

FIG 2. Axial source image from a $2 D$ TOF MR angiogram (A) demonstrates some loss of flow-related enhancement (arrow) associated with the web, indicative of diminished or absence of flow. Axial T1weighted fat-suppressed image $(B)$ through the level of the carotid bulb demonstrates $\mathrm{Tl}$ shortening (arrow) consistent with hemorrhage associated with the carotid web seen on the CTA.

a carotid web containing hemorrhage can be indistinguishable from hemorrhage in a nonstenosing atherosclerotic plaque. Whether carotid webs containing plaque hemorrhage confer a greater risk of ipsilateral ischemic events than webs without hemorrhage is unclear and requires future investigation.

In conclusion, we thank the authors for bringing the perhaps underdiagnosed entity of carotid web to our attention.

\section{REFERENCES}

1. Choi PM, Singh D, Trivedi A, et al. Carotid webs and recurrent ischemic strokes in the era of CT angiography. AJNR Am J Neuroradiol 2015 Jul 30. [Epub ahead of print] CrossRef Medline 
2. Morgenlander JC, Goldstein LB. Recurrent transient ischemic attacks and stroke in association with an internal carotid artery web. Stroke 1991;22:94-98 CrossRef Medline

3. Gupta A, Baradaran H, Schweitzer AD, et al. Carotid plaque MRI and stroke risk: a systematic review and meta-analysis. Stroke 2013;44: 3071-77 CrossRef Medline

4. Gupta A, Baradaran $\mathrm{H}$, Kamel H, et al. Intraplaque high-intensity signal on 3D time-of-flight MR angiography is strongly associated with symptomatic carotid artery stenosis. AJNR Am J Neuroradiol 2014;35:557-61 CrossRef Medline

(D).E. Lantos

(D).L. Chazen

(i) A. Gupta

Department of Radiology Weill Cornell Medical College New York, New York 